\title{
Heterogeneity of quaternary structure of glucosamine-6-phosphate deaminase from Giardia lamblia
}

\author{
Karolina Kwiatkowska-Semrau • Justyna Czarnecka • \\ Marek Wojciechowski • Slawomir Milewski
}

Received: 13 August 2014 / Accepted: 6 October 2014 / Published online: 19 October 2014

(C) The Author(s) 2014. This article is published with open access at Springerlink.com

\begin{abstract}
The oligoHis-tagged versions of glucosamine-6phosphate deaminase from Giardia lamblia (GlmNagB-HisN, Glm NagB-HisC) were constructed and purified to hear homogeneity, and their kinetic and structural properties were compared to those of the wild-type enzyme ( $G l m \mathrm{NagB}$ ). Introduction of the oligoHis tag at the Glm NagB C-terminus resulted in almost complete loss of the catalytic activity, while the catalytic properties of $G l m \mathrm{NagB}-\mathrm{HisN}$ and $G l m \mathrm{NagB}$ were very similar. The recombinant and wild-type enzyme exhibits heterogeneity of the quaternary structure and in solution exists in three interconvertible forms, namely, monomeric, homodimeric, and homotetrameric. Although the monomeric form is prevalent, the monomer/dimer/tetramer ratios depended on protein concentration and fell within the range from 72:27:1 to $39: 23: 38$. The enzyme is fully active in each of the oligomeric structures, efficiently catalyzes synthesis of $D$-glucosamine-6-phosphate from $D$-fructose-6-phosphate and ammonia, and its activity is not modified by GlcNAc6P, UDPGlcNAc, or UDP-GalNAc. GlcN6P deaminase of G. lamblia represents a novel structural and functional type of enzyme of the NagB subfamily.
\end{abstract}

Keywords Giardia - Glucosamine-6-phosphate deaminase . NagB · Quaternary structure

\section{Introduction}

NagB (glucosamine-6-phosphate deaminase; 2-amino-2-deoxy$D$-glucose-6-phosphate aminohydrolase (ketol-isomerizing);

K. Kwiatkowska-Semrau • J. Czarnecka • M. Wojciechowski •

S. Milewski $(\square)$

Department of Pharmaceutical Technology and Biochemistry,

Gdańsk University of Technology, 11/12 Narutowicza Str.,

80-233 Gdańsk, Poland

e-mail: slamilew@pg.gda.pl
EC 3.5.99.6, formerly EC 5.3.1.10) is in most organisms a catabolic enzyme and performs the isomerization and deamination reactions that transform $D$-glucosamine-6-phosphate (GlcN6P) to $D$-fructose-6-phosphate (Fru6P), with the concomitant release of ammonia. The reaction is basically reversible, but formation of GlcN6P by NagB is usually possible only at high substrate concentrations, so that under physiological conditions, the enzyme catalyzes a catabolic reaction (Calcagno et al. 1984). A notable exception to this rule is glucosamine-6phosphate deaminase of Giardia lamblia (syn. Giardia intestinalis, Giardia duodenalis), a flagellated unicellular protozoan, which is the common infective parasite of a number of vertebrates. G. lamblia lacks GlcN6P synthase, an enzyme responsible for GlcN6P formation in an overwhelming majority of organisms (Milewski 2002), and this task is accomplished by its NagB (Adam 2001).

GlcN6P deaminase is an ubiquitous enzyme and is absent only from several eukaryotic lineages and plants (ÁlvarezAñorve et al. 2011). Data accumulated so far indicate that there are three molecular versions of this enzyme, differing by quaternary structure and mechanisms of activity regulation. One of these versions, represented by Escherichia coli and mammalian $\mathrm{NagB}$, is a homohexameric protein, activated by an allosteric effector, $N$-acetyl-D-glucosamine-6-phosphate/ GlcNAc6P (Oliva et al. 1995; Arreola et al. 2003). On the other hand, GlcN6P deaminase of gram-positive bacteria including Bacillus subtilis and Streptococcus mutans is monomeric, and no physiological activator/inhibitor of this enzyme version has been identified (Vincent et al. 2005; Liu et al. 2008). Despite the structural and functional differences, both versions share a high homology of their primary sequences. A third type of protein demonstrating GlcN6P synthase activity was found in archea, has a homodimeric structure, demonstrates little homology to the mesophilic versions of NagB, but closely resembles the isomerase domain of GlcN6P synthase (Kim et al. 2007; Tanaka et al. 2005). 
GlcN6P deaminase of G. labmlia (GlmNagB) seems to be different from their already described and characterized counterparts in other organisms, due to its specific, crucial role in the life cycle and metabolism of the parasite. The cyst of G. lamblia is relatively inert, allowing prolonged survival for a long period under harsh environmental conditions, particularly in cold water, mostly due to the rigid structure of its cell wall composed of carbohydrates and proteins. A unique carbohydrate component of the cell wall is a polymer of $\mathrm{N}$-acetyl-D-galactosamine, synthesized in the inducible pathway, where GlcN6P deaminase catalyzes the first committed step. Although in G. lamblia, two genes encoding putative GlcN6P deaminase were identified, namely, GPI1 and GPI2, only the former is functionally expressed (van Keulen et al. 1998). Expression of the GPII gene (now called GN6PI) is $\sim 45$ times higher during encystment than the basal level (Eligio-García et al. 2011). A similar increase is observed for the intracellular GlcN6P level (Sener et al. 2004), which reflects enhanced GlcN6P deaminase activity. All these changes appear when Giardia trophozoites encyst in the presence of bile (Eligio-García et al. 2011). Interestingly, a similar phenomenon of differential expression of homologous genes was also noted for some other genes of G. lamblia, for example, those encoding glyceraldehyde 3-phosphate dehydrogenase (Yang et al. 2002). The anabolic role of GlcN6P deaminase and lack of GlcN6P synthase was also observed in the case of some other human pathogenic protozoans, like Entamoeba histolytica and Entamoeba invadens, containing chitin in their cyst cell walls (Aguilar-Díaz et al. 2010).

Structural information on G. lamblia GlcN6P deaminase is very limited. Steimle et al. (1997) described some basic data for the native enzyme purified to near homogeneity in a multistep procedure. Those data suggested a monomeric structure of the enzyme and presence of its two isoforms, differing by the $\mathrm{pI}$ values.

It was postulated that enzymes of cell wall biosynthesis pathways could be targeted by potential antiprotozoal drugs (Spindler et al. 1990). The anabolic GlcN6P deaminase was indicated as one of the potential targets for antiparasitic chemotherapy (Aguilar-Díaz et al. 2011), which was confirmed by results of the gene silencing experiments (Samanta and Ghosh 2012). It seems interesting therefore to find out a molecular basis of an apparent uniqueness of this enzyme. In this paper, we present results of our studies on construction, overexpression in E. coli, purification, and characterization of oligoHis-tagged versions of Glm NagBp.

\section{Materials and methods}

Bacterial strains and growth conditions

The E. coli TOP $10 \mathrm{~F}^{\prime}$ strain from Invitrogene was used in all cloning procedures. The $E$. coli BL21(DE3) pLysS strain from
Novagen was used for the overexpression of the wild-type Glm NagB and its oligoHis-tagged versions. Bacterial strains were cultured at $37{ }^{\circ} \mathrm{C}$ on LA solid medium $[1.0 \%(w / v)$ $\mathrm{NaCl}, 1.0 \%(w / v)$ tryptone, $0.5 \%$ yeast extract, and $1.5 \%(w /$ $v)$ agar] and in $\mathrm{LB}$ liquid medium $[1.0 \%(w / v) \mathrm{NaCl}, 1.0 \%$ $(w / v)$ tryptone, and $0.5 \%$ yeast extract] supplemented with $0.1 \mathrm{mg} / \mathrm{ml}$ ampicillin.

Plasmids, enzymes, and other materials

The pUC5-GlmNagB plasmid containing the GPII (GN6PI) gene cloned from the G. lamblia MR4 strain (Van Keulen et al. 1998) was a generous gift of Dr. Harry van Keulen, Department of Biological, Geological and Environmental Sciences, Cleveland State University, Cleveland, $\mathrm{OH}$. Other plasmids used were the following: pET15b (Novagen) and pETTopo from Champion pET Directional TOPO Expression Kits (Invitrogen). Restriction and modification enzyme DNA, molecular weight markers, and protein weight markers were purchased from Fermentas, Lithuania. DNA polymerase was from DNA-Gdańsk, Poland. T4 DNA ligase was from Epicentre.

Antibiotics, isopropyl- $\beta$-D-thiogalactopyranoside (IPTG), Fru6P, GlcN6P, and other reagents were purchased from Sigma. Purification of oligoHis-tagged proteins was performed on $\mathrm{Ni}^{2+}$-ion-dependent adhesion (IDA) agarose (His Bind Resin, Novagen). The wild-type enzyme was purified by ion exchange chromatography on the ResourceQ column.

DNA manipulations

Isolation of plasmid DNA was carried out according to the protocol of the Plasmid Mini kit (A\&A Biotechnology). DNA fragments were isolated from agarose gels following the standard procedure of the DNA Gel-Out kit (A\&A Biotechnology). DNA purification after enzyme treatment was performed according to the instructions in the DNA Clean-up kit (A\&A Biotechnology). DNA digestion with restriction enzymes was carried out according to the enzyme supplier's instructions. DNA fragments were ligated, and E. coli cells were prepared and transformed according to the standard methods (Sambrook et al. 1989).

Construction of bacterial expression plasmids

The recombinant expression plasmid encoding the wild-type GlcN6P deaminase of G. lamblia (pETTopo-GlmNagB) was constructed by PCR amplification from the pUC5-Glm NagB plasmid as a template, with the following primers: $5^{\prime}$-CACCATGCCGTCCATCCACGTC-3' (underlined sequence needed for further procedures) and 5'-GGATCCATTCACGTGT TTAAGCTTTTGC- $3^{\prime}$. The purified PCR product was then introduced into pETTopo using The Champion pET Directional TOPO Expression Kit (Invitrogen). 
The recombinant gene encoding G. lamblia GlcN6P deaminase with C-terminal His ${ }_{6}$ fusion (pETTopo-Glm NagB-HisC) was constructed by PCR amplification from the pUC5-GlmNagB plasmid as a template, with the following primers: $5^{\prime}$ CACCATGCCGTCCATCCACGTCTCC-3' (underlined sequence needed for further procedures) and 5'-CCTTAATGA TGATGATGATGATGAGCTTTTGCAGC-3' (sequence introducing hexaHis is bold).

The recombinant gene encoding G. lamblia GlcN6P deaminase with N-terminal $\mathrm{His}_{6}$ fusion (pET15b-Glm NagB-HisN) was constructed by PCR amplification from the pUC5-GlmNagB plasmid as a template. Addition of a 5'-NdeI restriction site was done using the forward primer (underlined sequence encodes restrictions sites of the enzyme) 5'-ATCACATATGCCGTCCATCCACGTCTCC-3', and a 3'-BamHI restriction site was generated using a reverse primer $5^{\prime}$-ATAGAGGAT CCATTCACGTGTTTAAGCTTTTGCAG-3'. The amplified DNA was digested with $N d e I$ and $B a m H I$ and inserted into the expression vector by ligation to the corresponding site of the NdeI/BamHI-digested pET15b vector.

The identity of recombinant plasmids was confirmed by restriction analysis and DNA sequencing.

\section{Protein expression}

E. coli BL21(DE3) pLysS cells, transformed with pETTopoGlmNagB, pETTopo-GlmNagB-HisC, or pET15b-GlmNagBHisN expression plasmid, were grown overnight in the LB liquid medium supplemented with ampicillin, at $37^{\circ} \mathrm{C}$. Sample of this culture $(10 \mathrm{ml})$ was then transferred to 11 of a fresh LB broth containing ampicillin, and cell suspension was grown at $37^{\circ} \mathrm{C}$. Expression was induced by the addition of $1 \mathrm{mM}$ IPTG to the cultures grown to $\mathrm{OD}_{600} \approx 0.5$ and incubation was continued for another $5 \mathrm{~h}$. Cells were harvested by centrifugation at $3,000 \times \mathrm{g}$ for $20 \mathrm{~min}$ at $4{ }^{\circ} \mathrm{C}$.

\section{Purification of the wild-type $\boldsymbol{G} \boldsymbol{I m} \mathrm{NagB}$}

The wild-type GlmNagB was purified by ion exchange chromatography. The bacterial pellet was suspended in buffer A (25 mM KH $\mathrm{KH}_{4} / \mathrm{K}_{2} \mathrm{HPO}_{4}$, pH 7.0, $0.5 \mathrm{mM}$ PMSF, $1 \mathrm{mM}$ DTT, $1 \mathrm{mM}$ EDTA), and the cells were disrupted by sonication $(3 \times 30$-s bursts with 30 -s intervals at a power setting of $30 \mathrm{~W}$, using a Branson sonifier 250) on ice. The lysate was centrifuged at $10,000 \times g$ for $20 \mathrm{~min}$ at $4{ }^{\circ} \mathrm{C}$. DNA was removed from the supernatant by addition of streptomycin sulfate solution to the final concentration of $1.1 \%$. The sample was stirred on ice during the entire procedure and 15-30 min afterward. The resulting mixture was centrifuged for $20 \mathrm{~min}$ $\left(10,000 \times g\right.$ at $\left.4^{\circ} \mathrm{C}\right)$, and the supernatant was collected. The extract after streptomycin sulfate precipitation was treated with a saturated ammonium sulfate solution to the final concentration of $60 \%$. The suspension was stirred on ice during the entire procedure and 15-30 min afterward and then centrifuged for $20 \mathrm{~min}\left(10,000 \times \mathrm{g}\right.$ at $\left.4{ }^{\circ} \mathrm{C}\right)$. The supernatant was discarded, and the precipitate was dissolved in $\sim 10 \mathrm{ml}$ of buffer A supplemented with $40 \mathrm{mM} \mathrm{NaCl}$ and $10 \mathrm{mM} \mathrm{MgCl}_{2}$. After complete dissolution, a $50 \%$ solution of polyethylene glycol 4,000 was added to a final concentration of $10 \%$. The suspension was stirred on ice during the entire procedure and 15-30 min afterward and then centrifuged for $20 \mathrm{~min}$ $\left(10,000 \times g\right.$ at $\left.4{ }^{\circ} \mathrm{C}\right)$. The supernatant was discarded, and the precipitate was dissolved in 5-10 $\mathrm{ml}$ of buffer A supplemented with $40 \mathrm{mM} \mathrm{NaCl}$ and $10 \mathrm{mM} \mathrm{MgCl}_{2}$. The solution was loaded on ResourceQ FPLC 6-ml column equilibrated with buffer B (20 mM KH $\mathrm{PO}_{4} / \mathrm{K}_{2} \mathrm{HPO}_{4}, \mathrm{pH}$ 7.0). The column was washed with $20-30 \mathrm{ml}$ of buffer $\mathrm{B}$, and elution was performed with a linear 0-1 $\mathrm{M} \mathrm{NaCl}$ gradient in buffer $\mathrm{C}(20 \mathrm{mM}$ $\mathrm{KH}_{2} \mathrm{PO}_{4} / \mathrm{K}_{2} \mathrm{HPO}_{4}, 1 \mathrm{M} \mathrm{NaCl} \mathrm{pH}$ 7.0). The elution rate was $5 \mathrm{ml} / \mathrm{min}$. Active fractions were pooled and concentrated by ultrafiltration using Amicon ${ }^{\circledR}$ Ultra-15" Centrifugal Filter Units $\left(10-\mathrm{kDa}\right.$ cut-off limit) at $2,500 \times \mathrm{g}$ at $4{ }^{\circ} \mathrm{C}$, until the protein concentration reached approximately $2 \mathrm{mg} / \mathrm{ml}$.

\section{Purification of oligoHis-tagged proteins}

GlmNagB-HisC and GlmNagB-HisN were purified by metal affinity chromatography. The purification of these recombinant proteins was performed similarly. The bacterial pellet was suspended in the buffer D $(20 \mathrm{mM}$ Tris/ $\mathrm{HCl}, \mathrm{pH} 8,500 \mathrm{mM}$ $\mathrm{NaCl}, 5 \mathrm{mM}$ imidazole, $0.1 \%$ Tween 20 , and $1 \mathrm{mM}$ phenylmethylsulfonyl fluoride/PMSF/), and the cells were disrupted by sonication $(3 \times 30$-s bursts with 30 -s intervals at a power setting of $30 \mathrm{~W}$, using a Branson sonifier 250) on ice. The lysate was centrifuged for $20 \mathrm{~min}\left(10,000 \times \mathrm{g}\right.$ at $\left.4{ }^{\circ} \mathrm{C}\right)$. The supernatant (crude extract) was applied to a $\mathrm{Ni}^{2+}$-IDA agarose column, pre-equilibrated with the buffer D. The column was then washed with the same buffer, followed by washing with the buffer E $(20 \mathrm{mM}$ Tris/ $\mathrm{HCl}, \mathrm{pH} 8,500 \mathrm{mM} \mathrm{NaCl}, 50 \mathrm{mM}$ imidazole, $0.1 \%$ Tween 20 , and $1 \mathrm{mM}$ PMSF). The oligoHistagged protein was eluted with buffer F containing $200 \mathrm{mM}$ imidazole (20 mM Tris/HCl, pH 8, $500 \mathrm{mM} \mathrm{NaCl,} 200 \mathrm{mM}$ imidazole, $0.1 \%$ Tween 20, and $1 \mathrm{mM}$ PMSF).

For further assays, the fraction collected was concentrated by ultrafiltration using Amicon ${ }^{\circledR}$ Ultra-15" Centrifugal Filter Units (10-kDa cut-off limit; Millipore, 2,500×g at $4{ }^{\circ} \mathrm{C}$ ) several times, until the protein concentration reached approximately $2 \mathrm{mg} / \mathrm{ml}$. The buffer was then exchanged for buffer A (25 mM KH $\mathrm{mO}_{4} / \mathrm{K}_{2} \mathrm{HPO}_{4}$, pH 7.0, $0.5 \mathrm{mM}$ PMSF, $1 \mathrm{mM}$ DTT, 1 mM EDTA) using the HiTrap ${ }^{\mathrm{TM}}$ Desalting Columns.

Enzyme activity determination

GlcN6P deaminase activity in both aminating and deaminating direction was assayed colorimetrically using a modification of the Elson-Morgan reaction. The standard reaction 
mixture for determination of aminating (GlcN6P synthesizing) activity contained the following: $40 \mu \mathrm{l}$ of $75 \mathrm{mM}$ Fru-6-P and $40 \mu \mathrm{l}$ of $1 \mathrm{M}$ ammonium chloride. The main component of a standard mixture for determination of deaminating activity was $40 \mu \mathrm{l}$ of $10-\mathrm{mM}$ GlcN6P solution. Compositions of the mixtures were supplemented by appropriate amounts of the enzyme preparation (depending on the concentration and activity of the protein preparation) and buffer A ( $25 \mathrm{mM}$ $\mathrm{KH}_{2} \mathrm{PO}_{4} / \mathrm{K}_{2} \mathrm{HPO}_{4}$, $\mathrm{pH}$ 7.0, $0.5 \mathrm{mM}$ PMSF, $1 \mathrm{mM}$ dithiothreitol (DTT), $1 \mathrm{mM}$ EDTA) in a final volume of $400 \mu \mathrm{l}$. The positive control contained $40 \mu \mathrm{l}$ of $100 \mathrm{mM}$ GlcN6P and $360 \mu \mathrm{l}$ of buffer $\mathrm{A}$, and the negative control contained $400 \mu \mathrm{l}$ of buffer A. The mixture was incubated at $37{ }^{\circ} \mathrm{C}$ for $30 \mathrm{~min}$. The reaction was stopped by $1-\mathrm{min}$ incubation at $100{ }^{\circ} \mathrm{C}$, and then, the mixture was cooled on ice. To acetylate GlcN6P present in the mixture, $200 \mu$ of saturated $\mathrm{NaHCO}_{3}$ solution and $100 \mu \mathrm{l}$ of $10 \%$ acetic anhydride in acetone prepared freshly before use were added; the mixtures were incubated at room temperature for $3 \mathrm{~min}$, then for another $3 \mathrm{~min}$ at $100{ }^{\circ} \mathrm{C}$ and finally cooled on ice. Subsequently, $200-\mu 1$ aliquots of $0.8 \mathrm{M} \mathrm{K}_{2} \mathrm{~B}_{4} \mathrm{O}_{7}(\mathrm{pH}$ 9.2-9.5) solution were added; the mixtures were boiled for $3 \mathrm{~min}$ and finally cooled on ice. Aliquots of the freshly prepared Elson-Morgan reagent ( $5 \mathrm{ml}$ of solution consisted of $1 \mathrm{~g}$ of 4dimethylaminobenzaldehyde, $100 \mathrm{ml}$ of glacial acetic acid, and $1.5 \mathrm{ml}$ of concentrated $\mathrm{HCl}$ ) were added, and the mixtures were incubated for $30 \mathrm{~min}$ at $37{ }^{\circ} \mathrm{C}$. Finally, absorbance of the samples at $585 \mathrm{~nm}$ was measured. One unit of specific aminating activity was defined as an amount of the enzyme that catalyzed the formation of $1 \mu \mathrm{mol}$ of GlcN6P/min/mg protein. One unit of specific deaminating activity was defined as an amount of the enzyme that catalyzed the breakdown of $1 \mu \mathrm{mol}$ of $\mathrm{GlcN} 6 \mathrm{P} / \mathrm{min} / \mathrm{mg}$ protein.

For determination of kinetic constants, enzyme activity was determined at variable $(0-10 \mathrm{mM})$ initial concentrations of appropriate substrates.

\section{Determination of native protein molecular mass}

Oligomeric structure of recombinant GlcN6P deaminase versions was studied, and molecular masses were determined by size exclusion chromatography (SEC) on Superdex 200HR 10/300 GL column. Protein was applied in the volume of $0.5 \mathrm{ml}$ and then eluted at $0.5 \mathrm{ml} / \mathrm{min}$ by $50-\mathrm{mM}$ potassium phosphate buffer ( $\mathrm{pH} 7.0$ ) containing $0.15 \mathrm{M} \mathrm{NaCl}$ (buffer G). Protein elution was followed at $280 \mathrm{~nm}$. The molecular mass standards were the following: dextran blue $(2,000 \mathrm{kDa})$, thyroglobulin $(669 \mathrm{kDa}), \beta$-amylase $(200 \mathrm{kDa})$, alcohol dehydrogenase $(150 \mathrm{kDa}), \mathrm{BSA}(66 \mathrm{kDa})$, and carbonic anhydrase $(29 \mathrm{kDa})$. The molecular masses of the proteins studied were calculated by interpolation of a plot of log molecular mass versus $K_{\mathrm{av}}$
The oligomeric structure was also analyzed by native PAGE using a NativPage ${ }^{\mathrm{TM}}$ System with Novex ${ }^{\circledR} 4-16 \%$ Bis-Tris gels kit (Invitrogen). The experiments were run according to the manufacturer's protocol.

Homology modeling of $\boldsymbol{G l m} \mathrm{NagB}$ and its oligoHis-tagged versions

Since the real structure of the $G \operatorname{lm} \mathrm{NagB}$ is not known so far, its hypothetical structure and those of the two oligoHis-tagged muteins were built by homology modeling. The modeling was based on the two templates: structure of the GlcN6P deaminase from Homo sapiens (pdbid: 1ne7) and structure of the enzyme from B. subtilis (pdbid: 2bkx). The amino acid sequences of these two enzymes are, respectively, 37 and $40 \%$ identical with the sequence of Glm NagB. The MODELLER9v2 software (Sali and Blundell 1993) was used for comparative modeling and VMD (Humphrey et al. 1996) for visualization.

\section{Other methods}

Protein concentration was determined by the Bradford method (1976). Discontinuous sodium dodecyl sulfatepolyacrylamide gel electrophoresis (SDS-PAGE) was performed by the method of Laemmli (1970), using a $5 \%$ stacking gel and a $15 \%$ separating gel.

\section{Results and discussion}

Cloning, expression, and purification of the recombinant proteins

Two overexpression plasmids containing DNA sequences encoding His $_{6}$-tagged $G l m \mathrm{NagB}$ were constructed. One of them encoding fusion of Glm NagB with N-terminus bound His $_{6}$-tag was obtained by cloning the $G \operatorname{lm} \mathrm{NagB}$ gene into the pET15 vector containing the $\mathrm{His}_{6}$-tag encoding sequence. Another plasmid containing fusion of Glm NagB with Cterminal $\mathrm{His}_{6}$-tag addition was obtained by PCR amplification, with one of the primers introducing the oligoHis-tag encoding sequence and subsequent introduction into the Champion $\mathrm{pET}$ Directional TOPO vector. Plasmid containing the gene encoding the wild-type GlcN-6-P deaminase of G. lamblia was constructed using the Champion pET Directional TOPO vector. The sequences of plasmids were verified by sequencing.

Conditions for overexpression were optimized in terms of temperature, inductor concentration, and time profile. The overproduction results were confirmed by SDS-PAGE of cell lysates. The best overexpression was obtained in E. coli 

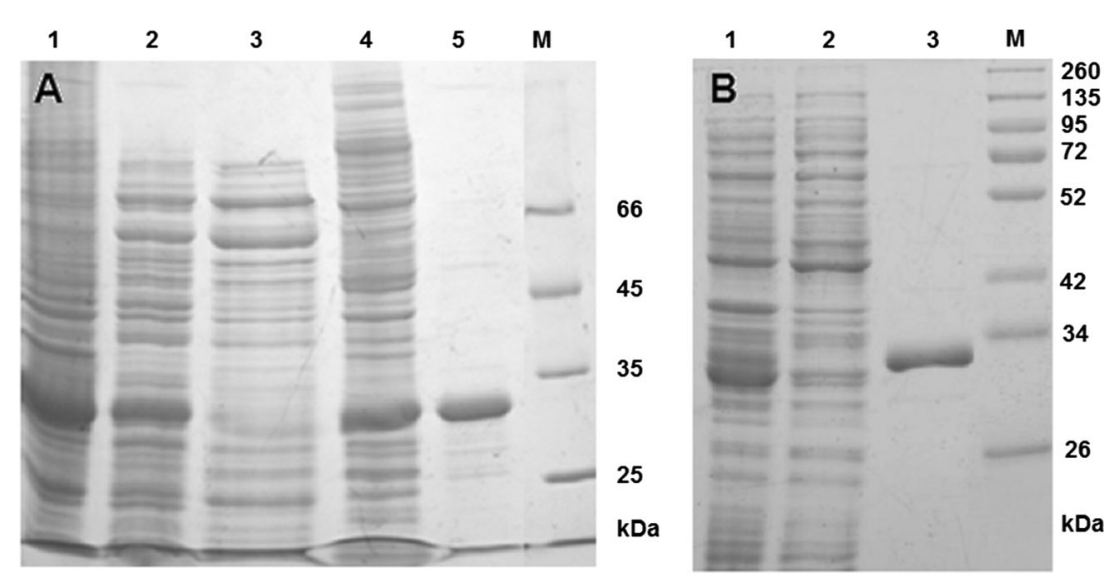

Fig. 1 SDS-PAGE analysis of purification of recombinant G. lamblia GlcN6P deaminase overexpressed in E. coli BL21(DE3) pLysS cells. a Wild-type Glm NagB; lane 1 - total lysate; lane 2-streptomycin sulfate precipitation; lane 3-ammonium sulfate precipitation; lane 4-PEG precipitation (dissolved sediment); lane 5-ion exchange chromatography on Resource Q; lane $M$-molecular mass markers. b Glm NagB-HisC; lane 1-total lysate; lane 2-wash of the $\mathrm{Ni}^{2+}$-IDA column with 0-50 mM imidazole; lane 3-200 $\mathrm{mM}$ imidazole eluate; lane $M$-molecular mass markers
BL21(DE3) pLysS cells, when expression was induced by addition of the IPTG $(1 \mathrm{mM})$ to the cultures grown to $\mathrm{OD}_{600} \approx 0.5$ and incubation was continued for another $5 \mathrm{~h}$. Densitometric analysis revealed that in each case, the recombinant protein constituted $8-24 \%$ of the total cytoplasmic protein pool (about $20 \mathrm{mg} / \mathrm{l}$ of the culture).

The purification of the wild-type GlmNagB from E. coli BL21(DE3) pLysS cells was a multistage process. Optimization of the purification process allowed obtaining a protein preparation with a purity of $98 \%$ with cumulative yield of $10.4 \%$ (Fig. 1a, Table 1). Although the yield was not high, it was much better than in the procedure described by Steimle et al., where it was lower than $1 \%$ (1997).

Glm NagB-HisC and Glm NagB-HisN were purified in a single chromatographic step using the $\mathrm{Ni}^{2+}$-IDA agarose resin. Unbound and weakly bound proteins were washed with 0 $50 \mathrm{mM}$ imidazole, and the recombinant protein was eluted with $200 \mathrm{mM}$ imidazole. The recombinant proteins were purified to near homogeneity ( $\sim 99 \%$, as revealed by a densitometric analysis) without considerable loss in protein yield. Effectiveness of purification was controlled by SDS-PAGE, and purity of the final preparations was estimated by densitometry of the CBB-stained gels. The results of the electrophoretic analysis of GlmNagB-HisC purification are shown in Fig. 1b. The SDS-PAGE profile for Glm NagB-HisN purification was apparently identical to that for Glm NagBHisC (not shown). Summary of purification of the wild-type Glm NagB and its $\mathrm{His}_{6}$-tagged muteins are shown in Table 2.

\section{Characterization of catalytic properties}

The purified wild-type Glm NagB and its $\mathrm{His}_{6}{ }_{-}$tagged versions were characterized in terms of enzymatic activity and molecular mass. The data are summarized in Tables 2 and 3. The specific activity and kinetic parameters of the wild-type enzyme were similar to that reported in literature (Steimle et al. 1997). Data obtained for the oligo-His tagged versions indicate that introduction of the tag at either $\mathrm{N}$ - or C-terminus affects enzyme activity, and in the case of Glm NagB-HisC, it resulted in almost complete loss of catalytic properties. The aminating (GlcN6P synthesizing) activity of Glm NagB-HisN was almost 1.5 times higher than that of the wild-type protein, while that of Glm NagB-HisC was two orders of magnitude lower. Substantial changes were noted for catalytic constants.

Table 1 Summary of the purification of GlmNagB overexpressed in Escherichia coli BL21(DE3) pLysS

\begin{tabular}{lllllll}
\hline Step & Volume [ml] & Total protein $[\mathrm{mg}]$ & Specific activity [U/mg] & Total activity [U] & Purification factor & Yield [\%] \\
\hline Crude extract & 20 & 360 & 8.6 & 3,096 & 1 & 100 \\
Streptomycin sulfate & 22 & 210 & 13.5 & 2,835 & 1.6 & 2.8 \\
Ammonium sulfate & 11 & 65 & 24 & 1,555 & 3.7 & 50.2 \\
PEG & 9 & 29.2 & 32 & 936 & 3.2 & 30.2 \\
Resource Q & 6 & 5.2 & 62 & 322 & 10.4 \\
\hline
\end{tabular}

The anabolic activity (synthesis of GlcN6P) was determined 
Table 2 The final purification results of wild-type GlmNagB and its muteins with $\mathrm{His}_{6}$-tagged at $\mathrm{C}$ - or $\mathrm{N}$-terminal

\begin{tabular}{lcc}
\hline Protein & Total protein $[\mathrm{mg} / \mathrm{l}]$ & Specific activity [U/mg] \\
\hline Glm NagB & 5.2 & 62.0 \\
Glm NagB-HisC & 3.2 & 0.5 \\
Glm NagB-HisN & 14.5 & 73.0 \\
\hline
\end{tabular}

Anabolic activity (synthesis of GlcN6P) was determined

The N-terminus modified enzyme exhibited about $30 \%$ higher $V_{\max }$ for the synthetic reaction than the wild-type Glm$\mathrm{NagB}$, while the respective constant for the catabolic reaction was unchanged. A substantial decrease of GlmNagB-HisC affinity to GlcN6P was found, reflected by its much higher $K_{\mathrm{M}}$ for this compound as a substrate in the catabolic reaction. A slightly higher $K_{\mathrm{M}}$ for Fru6P in the anabolic reaction was also noted for this mutein. Very low values of $V_{\max }$ of $\mathrm{Glm}$ $\mathrm{NagB}-\mathrm{HisC}$ in both reactions are the obvious consequence of the reduced activity. One may therefore conclude that catalytic properties of Glm NagB-HisN are similar to those of the wildtype GlmNagB, except for the slightly enhanced GlcN6P synthesizing activity. It is worth noting therefore that the introduced oligoHis-containing oligopeptide was quite long, as it contained 21 amino acid residues. On the other hand, introduction of a relatively small hexaHis fragment at the Cterminus resulted in a very substantial reduction of catalytic activity.

All recombinant versions of $G l m \mathrm{NagB}$ were also tested for a possible effect of GlcNAc6P, uridine 5'-diphospho- $N$-acetylD-glucosamine (UDP-GlcNAc), and uridine 5'-diphospho- $N$ acetyl-D-galactosamine (UDP-GalNAc) on activity. The first compound is known as an allosteric activator of EcoNagB (Calcagno et al. 1984) while the other two could be candidates for feedback inhibitors of the enzyme catalyzing the first committed step in the pathway of poly-GalNAc biosynthesis, by analogy to the similar role of UDP-GlcNAc, acting as a feedback inhibitor of eukaryotic GlcN6P synthase (Milewski 2002). However, in our hands, no changes in activity of

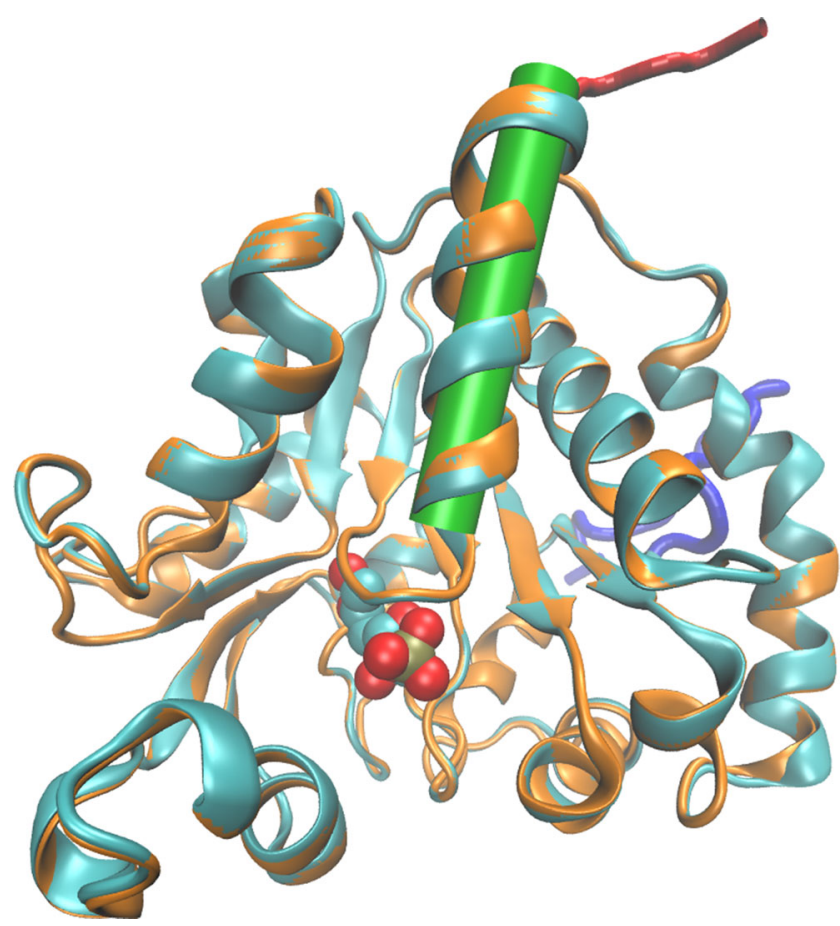

Fig. 2 Superimposed model structures of GlmNagB-HisC (orange) and Glm NagB-HisN (cyan) with their additional oligo-His fragments, colored red and blue, respectively. The substrate F6P (transferred from the template structure $2 \mathrm{bkx}$ ) is shown as a spacefill model bound in the active site. Location of the $\alpha 1$ helix is indicated by a green cylinder

Glm NagB were noted in the presence of any of the putative ligands at concentration $\leq 5 \mathrm{mM}$.

Molecular modeling of the enzyme

Since the 3D structure of Glm NagB and its oligoHis-tagged muteins is not known, a structural basis for the observed loss of catalytic activity upon introduction of the oligoHis tag at the $G l m \mathrm{NagB}$ C-terminus is not obvious. In an attempt to clear it up, the homology models of the enzyme and two muteins were built. Superimposed images of these models are shown in Fig. 2. Its is clear that both oligoHis tags in Glm NagB-HisC as well as in GlmNagB-HisN are located far from the active

Table 3 Summary of catalytic properties of the wild-type Glm NagB and its oligoHis-tagged versions

\begin{tabular}{|c|c|c|c|c|c|}
\hline \multirow[t]{3}{*}{ Enzyme version } & \multicolumn{5}{|c|}{ Kinetic parameters } \\
\hline & \multicolumn{3}{|c|}{ Aminating direction } & \multicolumn{2}{|c|}{ Deaminating direction } \\
\hline & $K_{\mathrm{M} \text { Fru6P }}[\mathrm{mM}]$ & $\mathrm{K}_{\mathrm{M} \mathrm{NH} 4 \mathrm{Cl}}[\mathrm{mM}]$ & $V_{\max }[\mu \mathrm{M} / \mathrm{min} / \mathrm{mg}]$ & $K_{\mathrm{M} \text { GleN6P }}[\mathrm{mM}]$ & $V_{\max }[\mu \mathrm{M} / \mathrm{min} / \mathrm{mg}]$ \\
\hline$G \operatorname{lm} \mathrm{NagB}^{\mathrm{a}}$ & $2.5 \pm 0.24$ & $19.0 \pm 1.90$ & $86.3 \pm 3.20$ & $0.38 \pm 0.16$ & $32.8 \pm 5.30$ \\
\hline Glm NagB & $2.8 \pm 0.14$ & $23 \pm 1.20$ & $100 \pm 5.20$ & $0.55 \pm 0.05$ & $38.1 \pm 1.70$ \\
\hline GlmNagB-HisN & $1.71 \pm 0.11$ & $14.0 \pm 0.80$ & $128.0 \pm 11.00$ & $0.95 \pm 0.15$ & $45.2 \pm 7.70$ \\
\hline GlmNagB-HisC & $3.52 \pm 0.15$ & $41.6 \pm 3.30$ & $2.0 \pm 0.16$ & $5.21 \pm 0.40$ & $1.21 \pm 0.11$ \\
\hline
\end{tabular}

${ }^{\text {a }}$ Reference data (Steimle et al. 1997) 
Fig. 3 a Results of SEC analysis of native Glm NagB on Superdex 200HR 10/300 GL column. $A U$ arbitrary units; Inside, b SDSPAGE analysis of fractions collected from SEC; c NativePAGE analysis of fractions collected from SEC. Lane Mmolecular mass markers; lanes 1 , 2 and 3 -fractions indicated in a

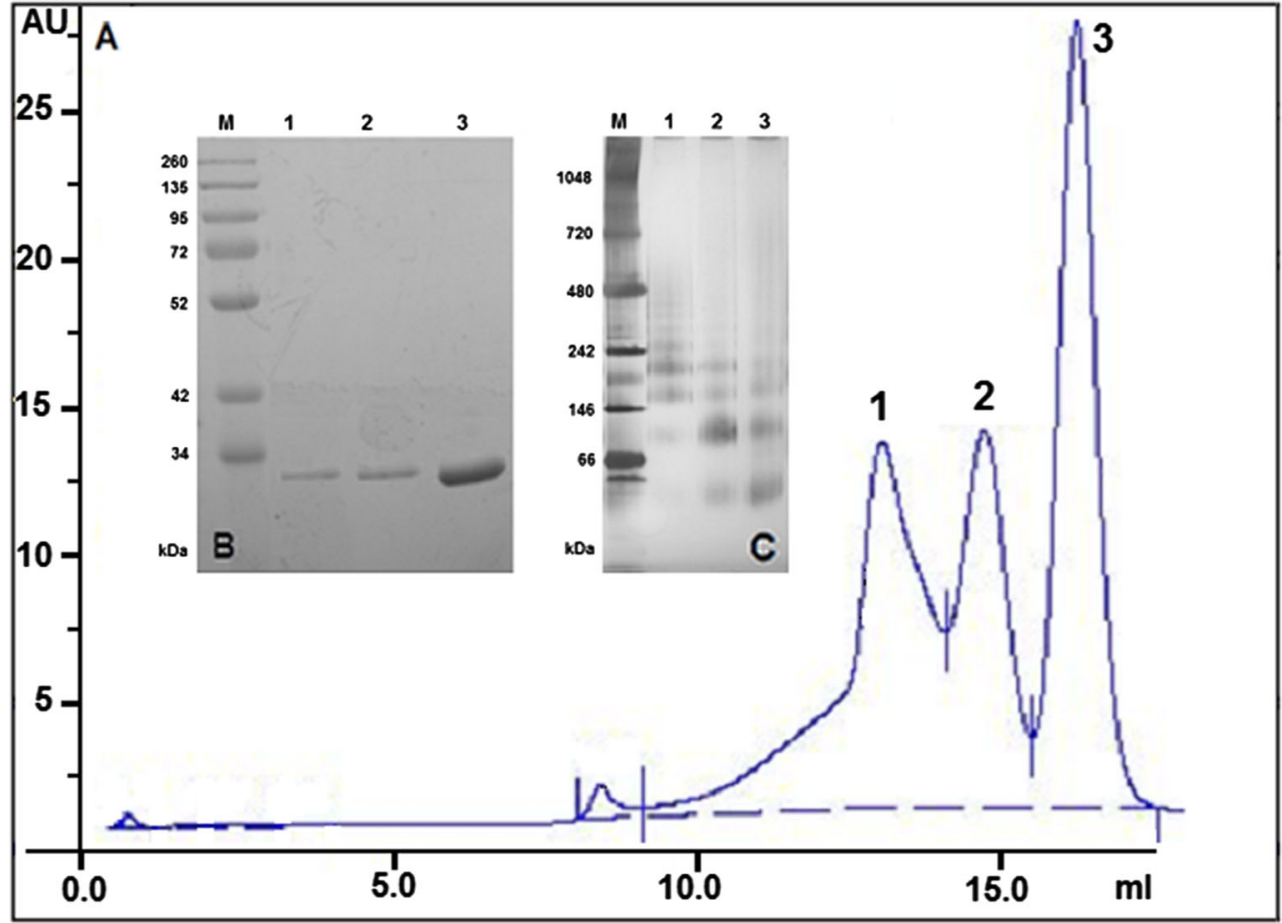

site; thus, they are not able to interfere with it directly. However, whereas the longer N-terminal fragment present in GlmNagB-HisN forms irregular loop at the site opposite to the active center, the shorter $\mathrm{C}$-terminal oligo-His fragment of Glm NagB-HisC is located close to the C-terminal part of the $\alpha 1$ helix. This helix, particularly its $\mathrm{N}$-terminal part, reach in Ser and Thr residues and containing conserved Gly36, corresponds to the analogous structure existing in all known structures of GlcN6P deaminases, which is directly involved in binding of the Fru6P or GlcN6P phosphate group and comprises common structural motif existing in various enzymes containing phosphate binding sites (Copley and Barton 1994). Preliminary molecular dynamics calculations (details not shown) revealed that the introduced C-terminal oligo-His fragment is possibly capping the C-terminal part of the $\alpha 1$ helix, thus interacting with it directly and affecting its dipole moment what in consequence may influence the substrate binding.

Determination of quaternary structure

SDS-PAGE analysis of GlmNagB, GlmNagB-HisN, and GlmNagB-HisC revealed presence of single bands migrating at $30.5 \pm 1.0,32.5 \pm 1.0$, and $32.0 \pm 1.0 \mathrm{kDa}$, respectively. These values are in a good agreement with theoretical molecular masses calculated for the appropriate amino acid sequences:

Table 4 Summary of results of SEC analysis of the wild-type Glm NagB and its oligoHis-tagged muteins

Fraction Native MW (SEC) [kDa] MW of the denatured protein (from SDS-PAGE) [kDa] Specific activity [U/mg] Putative quaternary structure

\section{Glm NagB}

1

2

3

Glm NagB-HisN

$\begin{array}{lcc}1 & 121.0 \pm 24.0 & 33.0 \pm 2.0 \\ 2 & 69.4 \pm 14.0 & 32.6 \pm 2.0 \\ 3 & 31.1 \pm 6.0 & 32.6 \pm 2.0 \\ \text { Glm NagB-HisC } & \\ 1 & 138.7 \pm 27.0 & 32.0 \pm 2.0 \\ 2 & 59.4 \pm 11.0 & 32.0 \pm 2.0 \\ 3 & 28.4 \pm 5.0 & 32.0 \pm 2.0\end{array}$

$30.7 \pm 2.0$

$30.7 \pm 2.0$

$30.7 \pm 2.0$

$58.0 \pm 11.0$
48

55

51

97

89

80

0.48

0.50

0.52
Homotetramer

Homodimer

Monomer

Homotetramer

Homodimer

Monomer

Homotetramer

Homodimer

Monomer 


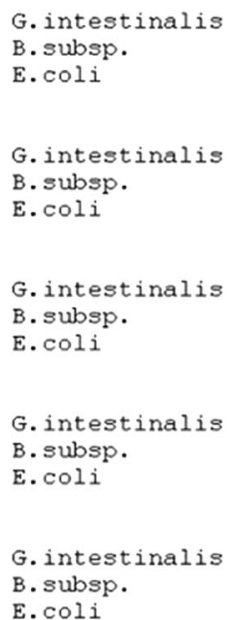

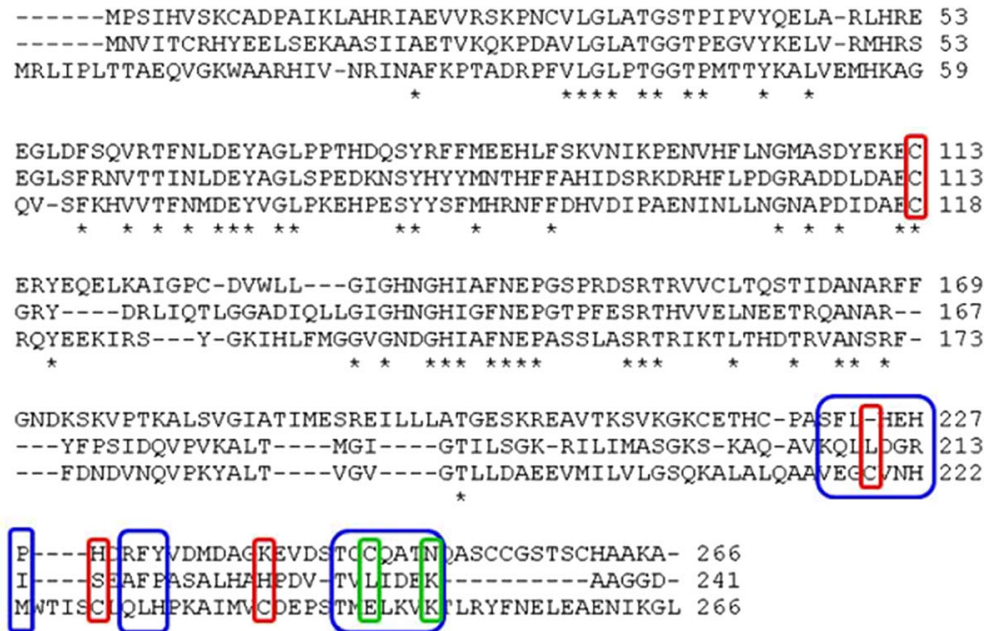

Fig. 4 Multiple alignment of amino acid sequences of glucosamine-6phosphate deaminase from Giardia intestinalis, Bacillus subsp., and Escherichia coli. Residues responsible for the formation of the hexameric structure of EcoNagB and their counterparts in Giardia and Bacillus proteins are in the blue frames. Glu246 and Lys250 which form a salt bridge linking a dimer of trimers in EcoNagB and their counterparts in Giardia and Bacillus proteins are in green frames. In red frames, the Cys residues that were supposed to be involved in disulfide bridges in $E$ coNagB and their counterparts in other enzyme versions
$29.4 \mathrm{kDa}$ for GlmNagB, $31.7 \mathrm{kDa}$ for GlmNagB-HisN, and $30.2 \mathrm{kDa}$ for GlmNagB-HisC (calculated by Compute $\mathrm{pI} /$ $\mathrm{MW}$ ). In contrast, multiple forms of Glm NagB and its oligoHis-tagged versions were found in SEC experiments. In all cases, three peaks corresponding to proteins of different sizes were detected (Fig. 3a, an exemplary SEC profile of the wild-type enzyme). Samples collected from respective fractions were analyzed for enzyme activity, effect of GlcNAc6P, UDP-GlcNAc, and UDP-GalNAc, by SDS-PAGE and by native PAGE. Results of these analyzes are shown in Table 4 and Fig. 3b, c. They showed that each of the enzyme versions occurred in three oligomeric forms, namely, monomeric, homodimeric, and homotetrameric. Oligomerization did not affect enzyme-specific activity, which was very similar for all the forms. None of the forms was activated/inhibited by GlcNAc6P, UDP-GlcNAc, or UDP-GalNAc. The relative content of oligomeric forms in analyzed samples was variable and depended mostly on protein concentration and storage period. The monomeric form was always dominant, but the monomer/dimer/tetramer ratios fell within the range from $72: 27: 1$ to $39: 23: 38$. The former ratio was characteristic for fresh protein preparations, obtained immediately after the purification process. The latter ratio was observed for preparations stored for several hours at $4{ }^{\circ} \mathrm{C}$ and for preparations with higher protein concentration. Interestingly, in samples of particular fractions from the SEC separation, containing a homogenous quaternary form of the enzyme, the other two forms were formed upon storage, with the whole system obviously going toward equilibrium. This tendency is also clear when looking at the results of native-PAGE analysis of fractions collected from SEC (Fig. 3c). Although in each of the lanes $1-3$, one of the bands is predominant, the other ones are also present, and this is likely a consequence of equilibration occurring in samples collected from SEC and stored for further native-PAGE analysis. Notably, the molecular masses calculated from the locations of the bands present in Fig. 3c are higher than the values expected for the monomer, dimer, or tetramer of $G l m \mathrm{NagB}(48,95,201 \mathrm{kDa})$, but similar deviations from the expected MWs have been reported previously for results of the native-PAGE analysis of other proteins, for example, fungal homocitrate synthase (Schöbel et al. 2010).

Results of the multiple structural alignment of Giardia, Bacillus, and E. coli GlcN6P deaminase shown in Fig. 4 clearly indicate that the residues 216-223, 230-232, and 244-250, identified as those responsible for the formation of the hexameric structure of EcoNagB (Oliva et al. 1995), have no counterparts of similar character in two other enzyme versions. Moreover, out of the four cysteinyl residues suspected to be involved in formation of intersubunit disulfide bonds in EcoNagB, namely, Cys118, Cys219, Cys228, and Cys239 (Altamirano et al. 1992, 1993; Horjales et al. 1992), only the first one is conserved in Glm NagB (Fig. 4). It is well understandable therefore why Glm NagB is not hexameric, despite the relatively high homology of GlmNagB and EcoNagB sequences (41\% identity). On the other hand, an amino acid sequence of $G l m$ NagB exhibits similar homology to those of the monomeric GlcN6P deaminase from $B$. subtilis (40\%) but lower to the dimeric NagB from Thermococcus kodakaraensis (25,1\%). Since NagB from archeons is homologous rather to the isomerase domain of GlcN6P synthase (ISOM) than to other NagBs from mesophilic organisms (Kim et al. 2007; Tanaka et al. 2005), it is obvious that Glm NagB is not similar to ISOM. The amino acid sequence of Glm NagB is homologous to that of GlcN6P deaminase of human 
pathogenic yeast Candida albicans encoded by the NAG1 gene (41\% identity), which is not activated by GlcNAc6P and supposed to be dimeric in the native form (Natarajan and Datta 1993), but this supposition has not been unequivocally confirmed yet. However, the GlcN6P deaminase of C. albicans is a catabolic enzyme, similarly as most of the representatives of the NagB subfamily, so that one cannot expect any close structural and functional analogy between these two enzyme versions.

The structural alignment reveals also a possible reason for the lack of an activating effect of GlcNAc6P on Glm NagB. In $E c o \mathrm{NagB}$, seven amino acid residues were identified as those directly involved in binding of the allosteric activator, namely, Tyr121, Asp141, Glu148, Ser151, Arg158, Lys160, and Tyr254 (Oliva et al. 1995; Vincent et al. 2005). Only four of them are conserved in Glm NagB, as Tyr116, Asp143, Ser146, and $\operatorname{Arg} 153$, respectively.

Due to the heterogeneity of its quaternary structure, GlmNagB may belong to the class of proteins known as morpheeins. The known examples include Pseudomonas aeruginosa GDP-mannose dehydrogenase (EC 1.1.1.132), mammalian CoA transferase (EC 2.8.3.5), and plant porphobilinogen synthase (EC 4.2.1.24) (Jaffe 2005). However, in the case of such protein, their oligomerization is one of the ways of activity regulation, and their oligomeric forms differ in activity and/or sensitivity to allosteric regulators. This seems not be the case for $G l m \mathrm{NagB}$, so that any functional reason for the observed heterogeneity of its quaternary structure is not known.

\section{Conclusions}

The oligoHis-tagged versions of $G \operatorname{lm} \mathrm{NagB}$ constructed and studied in this work demonstrate the same type of heterogeneity of quaternary structure as the wild-type protein, but the catalytic properties of Glm NagB-HisC are substantially different from those of Glm NagB-HisN and Glm NagB. Introduction of the hexaHis tag at the C-terminal of Glm NagB strongly reduces GlcN6P synthetic activity of the enzyme, while presence of the tag at $\mathrm{N}$-terminus has little if any effect. Apparent identity of kinetic parameters, insensitivity to the action of possible allosteric effectors (GlcNAc6P, UDPGlcNAc, and UDP-GalNAc) and existence in solution in three interconvertible quaternary forms (monomer, dimer, and tetramer) indicate that the easily isolable GlmNagB-HisN may serve as an adequate model of the wild-type enzyme in all structural studies.

Glm NagB is obviously different from other GlcN6P deaminases known so far, due to its dominant anabolic, not catabolic function, and the unique quaternary structure heterogeneity. There is little doubt therefore that $G \operatorname{lm} \mathrm{NagB}$ enzyme represents the fourth molecular version of enzymes of the NagB subfamily. Further, more detailed structural and mechanistic studies on this enzyme are highly desirable due to its potential as a putative target for antiprotozoal chemotherapy.

Acknowledgments The authors acknowledge the financial support of the Polish Ministry for Science and Higher Education, grant No N N301 105838. The generous gift of the pUC5-GlmNagB plasmid from Dr. Harry van Keulen, Cleveland State University, is gratefully acknowledged.

Open Access This article is distributed under the terms of the Creative Commons Attribution License which permits any use, distribution, and reproduction in any medium, provided the original author(s) and the source are credited.

\section{References}

Adam RD (2001) Biology of Giardia lamblia. Clin Microbiol 14:447475

Aguilar-Díaz H, Díaz-Gallardo M, Laclette JP, Carrero JC (2010) In vitro induction of Entamoeba histolytica cyst-like structures from trophozoites. PLoS Negl Trop Dis 4:e607

Aguilar-Díaz H, Carrero JC, Argüello-García R, Laclette JP, MoralesMontor J (2011) Cyst and encystment in protozoan parasites: optimal targets for new life-cycle interrupting strategies? Trends Parasitol 27:450-458

Altamirano MM, Plumbridge JA, Barba HA, Calcagno ML (1993) Glucosamine-6-phosphate deaminase from Escherichia coli has a trimer of dimers structure with three intersubunit disulphides. Biochem J 295:645-648

Altamirano MM, Plumbridge JA, Calcagno ML (1992) Identification of two cysteine residues forming a pair of vicinal thiols in glucosamine-6-phosphate deaminase from Escherichia coli and a study of their functional role by site-directed mutagenesis. Biochemistry 31:1153-1158

Álvarez-Añorve LI, Alonzo DA, Mora-Lugo R, Lara-González S, Bustos-Jaimes I, Plumbridge J, Calcagno ML (2011) Allosteric kinetics of the isoform 1 of human glucosamine-6-phosphate deaminase. Biochim Biophys Acta 1814:1846-1853

Arreola R, Valderrama B, Morante ML, Horjales E (2003) Two mammalian glucosamine-6-phosphate deaminase: a structural and genetic study. FEBS Lett 551:63-70

Bradford MM (1976) A rapid and sensitive method for the quantitation of microgram quantities of protein utilizing the principle of protein-dye binding. Anal Biochem 72:248-254

Calcagno M, Campos PJ, Mulliert G, Suástegui J (1984) Purification, molecular and kinetic properties of glucosamine-6-phosphate isomerase (deaminase) from Escherichia coli. Biochim Biophys Acta 787:165-173

Copley RR, Barton GJ (1994) A structural analysis of phosphate and sulphate binding sites in proteins: estimation of properties for binding and conservation of phosphate binding sites. J Mol Biol 242: 321-329

Eligio-García L, Maria del Pilar CV, Andrés FL, Apolinar CE, Adrian CC, Enedina JC (2011) Giardia intestinalis: expression of ubiquitin, glucosamine-6-phosphate and cyst wall protein genes during the encystment process. Exp Parasitol 127:382-386

Horjales E, Altamirano MM, Calcagno ML, Dauter Z, Wilson K, Garratt RC, Oliva G (1992) Crystallization and preliminary crystallographic studies of glucosamine-6-phosphate deaminase from Escherichia coli K12. J Mol Biol 226:1283-1286 
Humphrey W, Dalke A, Schulten K (1996) VMD: visual molecular dynamics. J Mol Graph 14:33-38

Jaffe EK (2005) Morpheeins - a new structural paradigm for allosteric regulation. Trends Biochem Sci 30:490-497

Kim KJ, Kim MH, Kim GH, Kang BS (2007) The crystal structure of a novel glucosamine-6-phosphate deaminase from the hyperthermophilic archaeon Pyrococcus furiosus. Proteins 68: 413-417

Laemmli UK (1970) Cleavage of structural proteins during the assembly of the head of bacteriophage T4. Nature 227:680-685

Liu C, Li D, Liang YH, Li LF, Su XD (2008) Ring-opening mechanism revealed by crystal structures of NagB and its ES intermediate complex. J Mol Biol 379:73-81

Milewski S (2002) Glucosamine-6-phosphate synthase- the multi-facets enzyme. Biochim Biophys Acta 1597:173-192

Natarajan K, Datta A (1993) Molecular cloning and analysis of the NAG1 cDNA coding for glucosamine-6-phosphate deaminase from Candida albicans. J Biol Chem 268:9206-9214

Oliva G, Fontes MRM, Garratt RC, Altamirano MM, Calcagno ML, Horjales E (1995) Structure and catalytic mechanism of glucosamine-6-phosphate deaminase from Escherichia coli at 2.1 Å resolution. Structure 3:1323-1332

Sali A, Blundell TL (1993) Comparative protein modeling by satisfaction of spatial restraints. J Mol Biol 234:779-815

Samanta SK, Ghosh SK (2012) The chitin biosynthesis pathway in Entamoeba and the role of glucosamine-6-P isomerase by RNA interference. Mol Biochem Parasitol 186:60-68
Sambrook J, Fritsch EF, Maniatis T (1989) Molecular cloning: a laboratory manual. Cold Spring Harbor Laboratory Press, Cold Spring Harbor

Schöbel F, Jacobsen ID, Brock M (2010) Evaluation of lysine biosynthesis as an antifungal drug target: characterisation of Aspergillus fumigatus homocitrate synthase and virulence studies. Eukaryot Cell 9:878-893

Sener K, Shen Z, Newburg DS, Jarroll EL (2004) Amino sugar phosphate levels in Giardia change during cyst wall formation. Microbiology 150:1225-1230

Spindler K-D, Spindler-Barth M, Londershausen M (1990) Chitin metabolism: a target for drugs against parasites. Parasitol Res 76:283-288

Steimle P-A, Lindmark D-G, Jarroll E-L (1997) Purification and characterization of encystment-induced glucosamine-6-phosphate isomerase in Giardia. Mol Biochem Parasitol 84:149-153

Tanaka T, Takahashi F, Fukui T, Fujiware S, Atomi H, Imanaka T (2005) Characterization of a novel glucosamine-6-phosphate deaminase from a hyperthermophilic archaeon. J Bacteriol 187:7038-7044

Van Keulen H, Steimle PA, Bulik DA, Borowiak RK, Jarroll E-L (1998) Cloning of two putative Giardia lamblia glucosamine 6-phosphate isomerase genes only one of which is transcriptionally activated during encystment. J Eukaryot Microbiol 45:637-642

Vincent F, Davies GJ, Brannigan JA (2005) Structure and kinetics of a monomeric glucosamine-6-phosphate deaminase. J Biol Chem 280: 19649-19655

Yang H-W, Yong T-S, Lee J-H, Im K, Park S-J (2002) Characterization of two glyceraldehyde 3-phosphate dehydrogenase genes in Giardia lamblia. Parasitol Res 88:646-650 